XX.

\title{
Eine norwegische Biertaufe: Probleme liturgischer Normierung im 13. Jahrhundert
}

\author{
Von
}

\section{Martin Kaufhold}

In den Tagen um das Fest des hl. Augustinus, den 28. August, des Jahres 1241 erhielt der Erzbischof von Trondheim negativen Bescheid aus Rom. Der Papst teilte dem nördlichen Metropoliten sein Urteil über eine Anfrage mit, deren Gehalt dem alten Kirchenvater in Afrika wohl einiges Stirnrunzeln bereitet hätte. Das päpstliche Schreiben war kurz und klar: Cum, sicut ex tua relatione didicimus, nonnunquam propter aque penuriam infantes terre tue contingat in cervizia baptizari, tibi tenore presentium respondemus, quod secundum doctrinam evangelicam oporteat eos ex aqua et spiritu sancto renasci, non debent reputari rite baptizati, qui in cervizia baptizantur $\left.{ }^{1}\right)$.

Eine interessante, wenn nicht gar eine unerhörte Begebenheit, wie es scheinen mag, die einen zweiten und nachforschenden Blick wert ist. Eine konventionell gestimmte Geschichtsschreibung würde in dem Vorfall wohl ein wüstes Wikingergelage vermuten, das die römische Disziplinargewalt völlig zu Recht auf den Plan rief. Eine moderne Untersuchung, die durch die kritische Schule interkultureller Kommunikation gegangen ist,

I) Diplomatarium Norvegicum (DN) I, ed. Ch. C. A. Lange/C. R. Unger, Christiania 1847, nr. 26; Potth., nr. 11048. Das Schreiben stammt vom 8. Juli 1241. Die Angelegenheit wurde an der Kurie im Zusammenhang mit einigen Anfragen des Erzbischofs von Trondheim, die insbesondere den Kreuzfahrerstatus seines Königs Håkon betrafen, erledigt: Potth, nr. 11045-11049. Die kuriale Post wurde dann wohl von einem Boten nach Norwegen zurückgebracht. Ein norwegischer Bote hatte bei einer vergleichbar dringlichen Mission 122545 Tage von Rom nach Trondheim gebraucht, nachdem sein Hinweg 50 Tage in Anspruch genommen hatte: Vgl. O. Springer, Medieval Pilgrim Routes from Scandinavia to Rome, Medieval Studies 12 (1950), 92-122, hier 122 . 
sollte ihr Augenmerk dagegen eher auf die Frage richten, ob die päpstliche Entscheidungsfindung hier nicht allzu unsensibel gegenüber der spezifischen kulturellen Situation der norwegischen christlichen Gemeinden gewesen ist. Es ließe sich zudem fragen, ob wir hier nicht ein frühes Beispiel jener auch kulturell geprägten vermeintlichen hegemonialen Überheblichkeit der römischen Kurie vor uns haben, die die Vertreter einer kritischen Kirche in den Entwicklungsländern noch in der Gegenwart beklagen ${ }^{2}$ ). Nicht zuletzt aber geht es um die Möglichkeiten und Bedingungen der Durchsetzung einer europäischen liturgischen Norm. Unternehmen wir einen Klärungsversuch.

Wir müssen mit einer Enttäuschung beginnen: Der Vorfall selbst läßt sich nicht mehr klären. Unsere Kenntnis über jenen befremdlich anmutenden Taufvorgang gelangt über die spröde Information aus dem päpstlichen Schreiben nicht hinaus. Einschlägige Archivanfragen ergaben, daß das Schreiben des Erzbischofs von Trondheim als verloren gelten muß. Es wäre wohl ohnehin nicht damit zu rechnen gewesen, daß er den Verlauf des Taufgeschehens ausführlicher geschildert hätte. Aller Spekulationen über den vermeintlichen Hergang wollen wir uns enthalten und unsere Aufmerksamkeit auf das liturgische Umfeld richten, das manchen Aufschluß bietet. Das Erzbistum Trondheim oder, wie es damals hieß, Nidaros, umfaßte seit seiner Einrichtung durch den Kardinallegaten Nikolaus von Albano im Jahre 1154 jene Diözesen, die die nördliche Randzone der Christenheit strukturierten: es waren fünf norwegische Bistümer, und neben den Färöern, den Hebriden und den Orkney-Inseln gehörten Island und schließlich Grönland dazu. ${ }^{3}$ )

2) Zum interessanten Thema interkultureller Kontakte vermag ich leider nur Beispiele zu nennen, die der neueren Geschichte entstammen: E. W. Said, Orientalism, New York 1979 und ders., Culture and Imperialism, New York 1993. Methodisch vergleichbare Studien, die das Problem kultureller Hegemonie an mittelalterlichen Beispielen studieren, sind mir nicht bekannt. Das Werk von J. L. Abu-Lughod, Before European hegemony: The world-system A. D. 1250-1350, New York u. a. 1989, ist schwerlich hilfreich. Es verbindet seine massive Schelte bisheriger Forschungserträge und Autoritäten mit einem völligen Verzicht auf jegliche Quellenstudien und trägt damit letztlich nur eine Reihe zu Recht vergessener Forschungsergebnisse vom Anfang des Jahrhunderts im neuen Gewande vor. Vgl. auch die Rezension von A. R. Lew is, in: Speculum 66 (1991), $605 \mathrm{f}$. $\mathrm{Zu}$ den Positionen der kritischen Kirche in der Diskussion um Evangelisierung und kulturelle Identität vgl. etwa: L. Boff, Gott kommt früher als der Missionar, Düsseldorf 1991.

3) Norges Gamle Love (NGL) I, Christiania 1846, 439-441; vgl. dazu: Ph. Zorn, Staat und Kirche in Norwegen bis zum Schlusse des Dreizehnten Jahrhunderts, München $1875,87-90$. Vgl. zu Grönland und Island, die in dieser Untersuchung wieder- 
Das Erzbistum Trondheim umfaßte damit die nördliche Peripherie der christlichen Welt ${ }^{4}$ ). So gab es manches randständige Problem. Die Grönländer waren aus Mangel an Priestern und Kirchen lange nicht in der Lage, ihren Toten ein christliches Begräbnis zu geben. So hatte man sich beholfen, indem man die Toten bei ihren Häusern in ungeweihter Erde begrub und die Grabstätten durch Pfähle kennzeichnete, die über der Brust des Bestatteten aus der Erde ragten. Kam ein Priester an den Ort, so zog man den Pfahl heraus und goß geweihtes Wasser in das Loch ${ }^{5}$ ). Der Mangel an Priestern mag mit dazu geführt haben, daß diejenigen, die dieses Amt versahen, offensichtlich noch in der zweiten Hälfte des 13. Jahrhunderts recht regelmäßig im Stande der Ehe lebten ${ }^{6}$ ). Diese Besonderheit des Zölibatsverständnisses am Polarkreis hat sogar das Interesse von Stefan Kuttner gefunden und ihn zu einem späten Aufsatz angeregt ${ }^{7}$ ). Neben diesen menschlichen Problemen aber gibt es weitere klare Hinweise darauf, daß die liturgischen Auflagen aus Rom den Seelsorgern vor Ort Probleme bereiteten. Es waren auch keine Bagatellprobleme, denn neben der Taufe, die unsere Frage erst auslöste, war auch das Sakrament der Eucharistie betroffen $\left.{ }^{8}\right)$. Hier standen die

holt angesprochen werden: H. Nottarp, Das Grönlandbistum Gardar, in: ZRG Kan. Abt. 50 (1964), 1-77; zur Legation des Kardinals Nikolaus und seiner kirchlichen Laufbahn - er wurde später als Hadrian IV. Papst - vgl.: R. B re yer, Die Legation des Kardinalbischofs Nikolaus von Albano in Skandinavien, Programm der städt. Realschule zu Halle/S. 1893; J. B a chmann, Die päpstlichen Legaten in Deutschland und Skandinavien 1125-1159, Berlin 1913, 113-121; E. M. Almedingen, The English pope (Adrian IV), London 1925, 78-123; W. Seegrün, Das Papsttum und Skandinavien bis zur Vollendung der nordischen Kirchenorganisation (1164), Neumünster 1167 (Quell. u. Forsch. z. Gesch. Schleswig-Holsteins 51), 146-166.

$\left.{ }^{4}\right) \mathrm{Zu}$ den geographischen Vorstellungen vgl. die verschiedenen Beiträge in dem von D. Unverhau und K. Schietzel herausgegebenen Sammelband: Das Danewerk in der Kartographiegeschichte Nordeuropas, Neumünster 1993.

${ }^{5)}$ Die Geschichte von Thorfinn Carlsemne, in Origines Islandicae, ed. G. Vifu sson/F. York Powell, Bd. II, Oxford 1905, 610-626, 617; vgl. H. S. Lucas, Medieval Relations between Flanders and Greenland, in: Speculum 12 (1937), 167-181, 177.

$\left.{ }^{6}\right) \mathrm{DN} \mathrm{I}, \mathrm{nr} .19$ (Gregor IX.) : ... abusus detestande consuetudinis inolevit, quod videlicet sacerdotes inibi existentes matrimonia contrahunt ...; ebenso Alexander IV.: Diplomatarium Islandicum I, Kaupmannahöfn 1857-76, nr. 151.

$\left.{ }^{7}\right)$ St. Kuttner, St. Jón of Holar: Canon Law and Hagiography in Medieval Iceland, in: Analecta Cracoviensia 7 (1976), 367-375; jetzt auch in: Ders., The History of Ideas and Doctrines of Canon Law in the Middle Ages, London 1980 (Var. Repr.).

$\left.{ }^{8}\right) \mathrm{Vgl}$. zu den Sakramenten: A. M. Landgraf, Dogmengeschichte der Frühscholastik III/1-2: Die Lehre von den Sakramenten, Regensburg 1954-55; J. Finkenzeller, Die Lehre von den Sakramenten im Allgemeinen: Handbuch der Dogmengeschichte IV I a, Freiburg-Basel-Wien 1980. 
Priester der Erzdiözese Trondheim mitunter vor dem Problem, daß sie das geforderte Brot und den Wein schlicht nicht zur Verfügung hatten. Auch hier hofften sie auf Bier: Cerevizia vel potus alius loco vini ${ }^{9}$ ).

Hier war das Problem im Grunde auch noch drängender. Denn, selbst, wenn wir annehmen möchten, daß die Taufe mit Bier aus akutem Wassermangel auch in der Erzdiözese Trondheim ein eher singuläres Ereignis darstellte, so war das eucharistische Problem ein grundsätzliches: cum vix aut nunquam vinum reperiatur in illis partibus ${ }^{10}$ ).

Die Kurie aber blieb hart. Die Eucharistie erfordere Brot und Wein, auch wenn die Umstände dies erschwerten. Ebensowenig durfte ein Christ mit Bier getauft werden, auch wenn es an Wasser mangelte. Aber worauf stïtze sich der Papst eigentlich?

Stünde die Kurie heute in einem ähnlich gelagerten Konflikt mit einer kritischen Gemeinde, so wäre ihre Position klar, und sie könnte auf die dogmatische Formulierung verweisen, die die Frage nach der Taufflüssigkeit eindeutig klärt: Die Materie dieses Sakraments ist wahres und natürliches Wasser, gleichgültig ob es kalt ist oder warm ${ }^{\prime \prime}$ ). Aber diese Formulierung aus dem Dekret für die Armenier stammt von 1439 und wurde auch dann erst allmählich verbindlich ${ }^{12}$ ). Eine dogmatische Orientierung stand dem Papst zum Zeitpunkt der norwegischen Anfrage nicht zur Verfügung ${ }^{13}$ ). Auch sonst gab es wenige verbindliche Regelungen, auf die sich eine Entscheidung berufen konnte. Wohl hatte sich das ältere Kirchenrecht dem Sakrament der Taufe zugewandt, die erörterten Probleme aber waren andere, grundsätzlichere gewesen: nach der heilsgeschichtlichen Einordnung des Sakraments, nach der Gültigkeit der Taufe durch schismatische Priester, nach der Kindertaufe $\left.^{14}\right)$. Daß bei diesen Taufhandlungen Wasser verwendet wurde, galt als selbstverständlich und wurde nicht eigens thematisiert ${ }^{15}$ ). Es gab noch

$\left.{ }^{9}\right)$ DN I, nr. 16. Die ganze Anfrage: Tue fraternitati querenti, an deficienti in quibusdam ecclesiis suffraganeorum tuorum eucharistia propter frumenti penuriam simplex oblata undecumque confecta populo, ut sub quadam decipiatur pietatis specie ... sint tradenda.

10) Ebd.; vgl. auch Nottarp, Grönlandbistum, 37

11) H. Denzinger/P. Hünermann, Kompendium der Glaubensbekenntnisse und kirchlichen Lehrentscheidungen, 37. Aufl. Freiburg/Br. u. a. 1991, 1314.

12) Ebd., S. 453; das ganze Dekret ebd, nr. 1310-1328.

13) Vgl. etwa Landgraf, Dogmengeschichte III/1, 210: „Einer der Gründe unserer Unklarheit liegt darin, daß man in jener Zeit nichts besaß, was unserem heutigen dogmatischen Traktat De sacramentis in genere entspräche."

$\left.{ }^{14}\right)$ Decretum Gratiani, De cons., d. IV.

15) Z. B.: Decretum, De cons., d. IV, c. 1 : Renascitur homo ex aqua, visibili sacra- 
keinen drängenden Klärungsbedarf. Der theologische Weg, der schließlich auch die Materie des Sakraments in die Betrachtung miteinbezog, war die Entwicklung eines Verständnisses von der Taufe und schließlich von den Sakramenten allgemein als zusammengesetzten Zeichen der göttlichen Gnade $^{16}$ ). Die Theologie trug damit der Herausforderung Rechnung, daß die Taufe nach christlichem Verständnis von Anfang an eine symbolische Abwaschung der Sünden und eine Wiedergeburt im Heiligen Geist bewirkte, die unerläßlich war für die Erlangung des ewigen Lebens: Nisi quis renatus fuerit ex aqua et Spiritu non potest introire in regnum Dei, so hatte Jesus dem Nikodemus in der Version der Vulgata geantwortet (Joh. 3, 5).

Als Augustinus zu Beginn des 5. Jahrhunderts seine Traktate über das Johannesevangelium verfaßte, lenkte er die Aufmerksamkeit auf die einzelnen Komponenten des Taufsakraments. Seine Frage galt dem Verhältnis von segnendem Wort und dem Wasser der Taufe, und er gab einen Impuls für die Auseinandersetzung mit dem Sakrament, der zwar erst sehr viel später aufgenommen wurde, aber letztlich durch eine Präzisierung des Problems das Nachdenken über die Materie des Sakraments erst auf den Weg brachte. ${ }^{17}$ )

Tatsächliche Wirkung entfaltete die weiter entwickelte Theorie von der Zusammensetzung der Sakramente jedoch erst im Gefolge der Rezeption des Sentenzenwerkes von Petrus Lombardus, das zu dem Lehrbuch des aufstrebenden Schul- und Universitätsbetriebes wurde ${ }^{18}$ ). Petrus hatte ohne allzuviel eigenes Zutun im vierten Buch seines Sentenzenwerkes durchaus zeitgenössische Vorstellungen aufgegriffen und zu den Sakramenten knapp angemerkt: Duo autem sunt in quibus sacramentum consistit, scilicet verba et res; verba, ut invocatio Trinitatis; res, ut aqua, oleum et huiusmodi ${ }^{19}$ ). Mit

mento, et spiritu, invisibili scilicet intellectu, ut simbolum baptismi visibiliter accipiat, et spiritualem intellectum ipsius simboli percipiat. Vel ex aqua visibili et spiritu sancto (Augustinus).

16) Vgl. dazu mit Blick auf die für den hier untersuchten Zeitraum relevanten Texte: D. van den Eynde, The Theory of the composition of the sacraments in early Scholasticism (1125-1240), in Franciscan Studies 11 (1951), 1-10, 117-144 und 12 (1952), 1-26.

$\left.{ }^{17}\right)$ Aur. Augustinus, In Johannis evangelium tract. 80, 3, Migne P. L. 35, col. 1840 : Detrahe verbum, et quid est aqua nisi aqua? Accedit verbum ad elementum, et fit sacramentum. "These words were to play a big part in the composition of the sacraments": van den Eynde, Theory FS 11, 4. Zu Augustinus vgl. etwa P. Brown, Augustinus of Hippo, London 1967.

18) Van den Eynde, Theory FS 12, 1; zu Petrus Lombardus vgl.: M. L. Colish, Peter Lombard, Bd 1-2, Leiden-New York-Köln 1994 (Brill's studies in intelectual history 41/1-2).

19) Sententiae, L. IV. d. I c. 5: Magistri Petri Lombardi Sententiae in IV Libris Di- 
entsprechender Verzögerung begann das Grundschema von res/materia und verba seit den dreißiger Jahren des 13. Jahrhunderts nun die Kategorien der Auseinandersetzung mit den Sakramenten zu prägen. Die Anfrage der Norweger von 1241, ob bei Wassermangel auch mit Bier getauft werden dürfe, fiel also genau in die Phase einer eröffneten Diskussion um die Zusammensetzung der Sakramente ${ }^{20}$ ).

Die Frage nach der Materie der Sakramente - de differentia materiae in sacramentis - fand zuerst die Aufmerksamkeit des englischen Franziskanertheologen Alexander von Hales. Seine Behandlung der Frage im vierten Buch seiner Glossa in Libros Sententiarum ist die erste Erörterung dieser Art. ${ }^{21}$ )

Einen Höhepunkt erreichte die Sakramententheologie dann bei Thomas von Aquin $^{22}$ ). In einem umfangreichen und dabei noch unvollständig gebliebenen Sakramententraktat im dritten Buch der Summa Theologiae legte Thomas sein Verständnis dar ${ }^{23}$ ). Der Traktat entstand wohl in Thomas' letzten Lebensjahren zu Beginn der 70er Jahre ${ }^{24}$ ). Seine Entstehungszeit liegt damit noch im Zeithorizont unserer Untersuchung, auch wenn der Traktat natürlich die negative kuriale Antwort von 1241 nicht verursacht haben kann. Hier geht es um eine Prüfung der zeitgenössischen Argumentation. Die verschiedenen Meinungen finden einen Niederschlag bei Thomas, der sie ohne revolutionäre Absicht erörterte und mit seinem praktischen Sinn prüfte. Ein knappes Meinungsbild zu erstellen, ist anhand der Argumente

stinctae II, 3. Aufl. Grottaferrata 1981, 235 (Spic. Bonav. V); zu Petrus' Sakramentenverständnis: Colish, Peter Lombard 2, 516-698, zur Taufe : 532-48.

20) Vgl. mit den entsprechenden Textbeispielen: van den Eynde, Theory FS 12 , $1-26$.

${ }^{21)}$ Magistri Alexandri de Hales Glossa in quattuor libros Sententiarum Petri Lombardi IV, d. XXVI, 5, Quaracchi 1957, 450-55 (Bibl. Francisc. Schol. Med. Aevi XV), Vgl. van den Eynde, Theory FS 12, 8-12.

22) $\mathrm{Zu}$ Thomas vgl. einführend: O. H. Pesch, Thomas von Aquin, Grenze und Größe mittelalterlicher Theologie, 2. Aufl. Mainz 1989 und J. P. Torrell, Magister Thomas, Leben und Werk des Thomas von Aquin, Freiburg-Basel-Wien 1995.

${ }^{23}$ ) Summa theologiae (S. th.) III, q. 60-90, Taufe: 66-71; Sancti Thomae Aquinatis Opera Omnia iussu Leonis XIII P. M. edita, Bd. 12, Rom 1906; vgl. zu Thomas' Sakramentenlehre: Pesch, Thomas, 343-373; H.-F. Dondaine, La définition des sacrements dans la somme theologique, in: RSPhTh 31 (1947), 213-228; Vgl. auch: E. Schillebeckx, De sacramentale heilseconomie, Theologische bezinning op S. Thomas' sacramentenleer in het licht van de traditie en van den heddendagse sacramentsproblematik, Antwerpen 1952.

${ }^{24}$ ) J. A. Weisheipl, Friar Thomas d'Aquino. His Life, Thoughts and works, Oxford 1975, 298-320; Torrell, Magister Thomas, $347 \mathrm{f}$. 
von Alexander von Hales bis zu Thomas für unseren Zweck hinreichend möglich. Das meiste bietet ohnehin Thomas.

Für den Dominikaner Hugo von St. Cher (gest. 1263), der sich in Paris mit den Problemen des Sakramentsverständnisses befaßte und der der theologischen Diskussion bedeutende Impulse gab ${ }^{25}$ ), war die Sache klar: getauft werden konnte nur mit Wasser - in alio liquore non datur gratia baptisma$l i s^{26}$ ). Denn nur das Wasser habe durch die Berührung mit der Hostie die materielle Kraft, die Wiedergeburt des Getauften zu bewirken ${ }^{27}$ ). Parallel argumentierte Hugo in Hinblick auf die Eucharistie: allein das Weizenbrot erfülle die Voraussetzungen für die Transsubstantiation ${ }^{28}$ ). Vom Wein sprach Hugo an dieser Stelle nicht, aber nicht, weil es sich damit anders verhielt, sondern wohl, weil er gar nicht auf die Idee kam, daß der Wein in der Eucharistie fraglich sein könne. Er machte im Anschluß auch sofort klar, daß er die materielle Beschaffenheit von Brot und Wein im Auge hatte ${ }^{29}$ ). Das war klar, grundsätzlich und wenig hilfreich für einen Missionsbischof auf Grönland.

Daß dessen Probleme den Theologen zwar bewußt waren, aber im traditionellen Normensystem lösbar erschienen, zeigt die Argumentation des Thomas von Aquin. Der Doctor angelicus war ein durchaus praktischer Mann. Er ging von der Funktion der Taufe aus: der Abwaschung der Sünden. So fragt er funktional nach dem Charakter der Flüssigkeit, die man hierzu zweckmäßig verwende. Dadurch gelangten auch Alternativen in den Horizont der erörterten Möglichkeiten: sed multa alia sunt ablutiva quam aqua: sicut vinum et oleum et alia huiusmodi ${ }^{30}$ ). Dennoch verwarf Thomas Öl oder Wein - und damit auch Bier, denn im Gegensatz zum Wasser ließen diese Flüssigkeiten Verunreinigungen zurück - illa etiam non ita communiter et abundanter habentur sicut aqua ${ }^{31}$ ).

Die Frage nach anderen Substanzen, die ebenfalls bei dem Taufritus Verwendung fänden, klärte er durch eine deutliche Unterscheidung ${ }^{32}$ ). Es gäbe

\footnotetext{
25) Vgl.van den Eynde, Theory FS 12, 12-22.

26) Die betreffenden Passagen aus den Quaestionen Hugos sind nicht ediert. Sie sind überliefert in Vatik. lat. 1098, hier f. 143d-144a. Die zitierte Passage hier nach van den Eynde, Theory FS 12, 15.

27) Ebd.

${ }^{28}$ ) Ebd. : ... ita sub alia forma quam forma panis triticei non datur Corpus Christi.

29) Ebd.

$\left.{ }^{30}\right)$ S. th. III, q. 66, art. 3. (2).

${ }^{31)}$ Ebd.- ad 2.

32) S. th. III, q. 66, art. 10. (2).
} 
beim Taufsakrament eben Bestandteile, die ihm wesentlich seien und andere, die eher einen feierlichen Begleiteffekt hätten, so die Salbung mit Öl und Chrisma ${ }^{33}$ ). Thomas ging auch der Frage der Qualität des Wassers nach ${ }^{34}$ ). Denn er sah wohl, daß die Forderung nach reinem Wasser für die Taufe an praktische Grenzen stie $\beta$, insofern als das verfügbare Wasser aus den Flüssen oder dem Meer allerlei Beimischungen enthalte und daher nicht uneingeschränkt als aqua simplex gelten könne ${ }^{35}$ ). Wenn also grundsätzlich Beimischungen zum Taufwasser in Kauf genommen werden mußten, ließe sich doch auch mit Rosenwasser und dem Wasser, das die Alchimisten in ihren Experimenten gewönnen, taufen - und warum dann nicht auch mit Wein $\left.^{36}\right)$ ?

Thomas verwirft alle diese Möglichkeiten und unterscheidet zwischen Beimengungen, die aus einem natürlichen Prozeß hervorgegangen seien, und dem Resultat eines künstlichen Vorgangs. Es bleibt beim reinen Wasser, aber die Diskussion hatte doch für eine kulturgeschichtliche Fragestellung erheblich an Problemwahrnehmung gewonnen. Thomas sah auch, daß der Mangel an Wasser das geforderte Verfahren der Abwaschung erheblich erschweren konnte ${ }^{37}$ ). Doch auch dann durfte sich der Priester nicht mit Bier behelfen, sondern mußte sein Taufwasser sparsam versprengen ${ }^{38}$ ).

Thomas ging also keine neuen Wege, aber er sah durchaus die neuen Probleme, und seine Antwort war die eines Mannes, der in einer Welt des Handels großgeworden war. Denn die Lösung des Konflikts zwischen der Universalität der Kirche und ihrer Sakramente und den verschiedenen kulturellen Eigenheiten in dieser einen Kirche sah er im Handel. Anläßlich der Erörterung der Materie des Altarsakramentes stellte er fest: ... licet non in omnibus terris nascatur triticum et vinum, tamen de facili ad omnes terras deferri potest quantum sufficit ad usum huius sacramenti ${ }^{39}$ ).

Auf den Weinhandel mit Norwegen werden wir bald zurïckkommen, nicht jedoch, ohne vorher noch eine Stimme zu hören, die den liturgischen Problemen des Polarkreises näher war. Albert der Große verfaßte nur wenige Jahre nachdem die Frage nach der Rechtmäßigkeit der Biertaufe in

$\left.{ }^{33}\right)$ Ebd. (ad 2).

$\left.{ }^{34}\right)$ S. th. III, q. 66, art. 4.

35) Ebd. (1).

${ }^{36}$ ) Ebd. (5) und (ad 5).

${ }^{37}$ ) S. th. III, q. 66, art. 7. (c): Et ideo, quamvis tutius sit baptizare per modum immersionis, quia hoc habet communior usus: potest tamen fieri baptismus per modum aspersionis ... Et hoc praecipue propter necessitatem ... potest imminere necessitas propter paucitatem aquae.

38) Ebd.

$\left.{ }^{39}\right)$ S. th. III, q. 74, art. 1. (ad 2). 
Rom verneint worden war, eine eigene Schrift über die Sakramente, die auf seinen öffentlichen Disputationen als Magister an der Universität Paris basierte $\left.{ }^{40}\right)$. Darin findet sich für unsere Fragestellung Interessantes: der Frage einer möglichen Biertaufe wird ein subtiler Blick zuteil ${ }^{41}$ ). Es war das nämliche Problem, das auch Thomas zur Einbeziehung der alkoholischen Getränke in seine Erwägungen veranlaßt hatte. Auch Albertus fragte, ob man jegliches Wasser zur Taufe verwenden dürfe, aber der doctor universalis gibt eine positivere Antwort, denn für die Taufe sei Wasser erforderlich, und dessen Natur sei durch keinen menschlichen Eingriff zu verändern ${ }^{42}$ ). Ergo videtur, quod in cervisia et medone et talibus possit fieri baptismus ${ }^{43}$ ). Damit war der Dialektik einer Disputation noch nicht Genüge getan, aber die vermittelnde Lösung, die Albertus schließlich formulierte, hätte dem norwegischen Anliegen einigen Spielraum gelassen. Denn Albertus verlangte, bei gemischten Flüssigkeiten den Grad der Mischung zu bedenken. Sei er derart, daß der Wasser-Charakter eindeutig überwiege, dann könne mit der Flüssigkeit getauft werden. Sei aber die beigemischte Materie gegenüber dem Wasser dominant, so sei die Lösung ungeeignet. Dies gelte zumeist für Bier, und daher sei eine Taufe mit Bier in der Regel ausgeschlossen ${ }^{44}$ ). Das war kein grundsätzlicher Ausschluß. Die Norweger hätten Albertus überzeugen können, wäre ihr Bier nur wässrig genug gewesen.

Den Papst überzeugten sie nicht. Dabei hatten sich die nordischen Bistümer bei diesem gesamten Problemkomplex große Mühe gegeben und kaum etwas unversucht gelassen, um in ihren Regionen etwa eigenen Wein zu gewinnen. Der grönländische Bischof Jon Smyrill Amasson (1187-1209) ${ }^{45}$ )

$\left.{ }^{40}\right)$ De sacramentis, in: Alberti Magni Opera omnia 26, Münster 1958. Zur Datierung der Pariser Jahre des Albertus vgl. etwa J. A. We i sheipl, The Life and works of St. Albert the Great, in: Ders.(Hg.), Albertus Magnus and the Sciences, Commemorative essays, Toronto 1980 (Studies and texts 49), 13-51, 22.

$\left.{ }^{41}\right)$ De Sacr. tract. III, de bapt., q. 1, art. 2.

${ }^{42}$ ) Ebd. (4): ... tunc quaeritur, an in omni aqua. Et videtur, quod sic, quia artificium non mutat speciem ...

$\left.{ }^{43}\right)$ Ebd.

${ }^{44)}$ Ebd. (ad 5): Ad id, quod quaeritur de aqua mixta, dicunt quidam, quod in mixta potest fieri baptismus, verbi gratia, in cervisia et aliis similibus. Sed nobis videtur considerandum in mixtione. Si enim natura aquae dominans est et remanens species, quod non proprie dicitur mixtum, videtur nobis, quod possit fieri baptismus. Si autem ipsa mixta recesserunt a naturis propriis in materiam mediam, tunc puto, quod non possit fieri baptismus, quia mixtum nullum est miscibilium, et hoc magis videtur in cervisia et medone.

${ }^{45}$ ) Grönlands Historiske Mindesmaerker (GHM) 3, Kopenhagen 1845, ND Kopenhagen 1976,896 . 
brachte 1203 die Kenntnis der Weingewinnung aus Beeren nach Island ${ }^{46}$ ). Der Bischof reiste alsbald weiter nach Rom, aber eine gute Beerenernte im Sommer hielt das Interesse wach, und das Ergebnis soll gut gewesen $\operatorname{sein}^{47}$ ).

Bischof Jon Smyrill hatte das Rezept der Beerenvergärung von seinem König Sverri (1177-1202) erlernt ${ }^{48}$ ). Der König war kein Freund des Alkoholhandels, dessen Probleme natürlich die Kehrseite der zwei Generationen später von Thomas vertretenen Handelslösung darstellten. Deren Schattenseiten waren schon am Ende des 12. Jahrhunderts hervorgetreten. In einer Rede in Bergen 1186 hatte König Sverri sich an die Handelspartner Norwegens gewandt, er hatte den Engländern für ihre Weizenimporte gedankt und auch die Kaufleute von den Orkneys, Färöern und aus Island seiner Wertschätzung versichert. Dann aber war er zu den Deutschen gekommen, die das Land durch ihre Käufe von Butter und Dorsch schädigten:

Als Gegenwert haben sie Wein hier eingeführt, den die Leute gekauft haben, sowohl die Mannen meines Gefolges als die Bürger und Kaufleute. Aus diesem Kauf ist viel Böses entstanden und nichts Gutes. Viele haben deswegen ihr Leben verloren, und einige die Glieder. Manche tragen Schaden davon für die ganze Zeit ihres Lebens. Andere haben Schimpf erlitten, sind verwundet oder geprïgelt worden, und das kommt alles von der allzu großen Trinkerei $\left.{ }^{49}\right)$.

Ein zeitgenössischer dänischer Autor bestätigt diesen Eindruck von Bergen als einer internationalen Handelsstadt mit lebendiger Atmosphäre, in der es aber immer wieder zu bedrohlichen Alkoholexzessen komme ${ }^{50}$ ).

${ }^{46}$ ) Póls Saga, Origines Islandicae I (wie Anm. 5), 502-34, 515f.; GHM 3, 9: Vin af Baer; vgl. auch Nottarp, Grönlandbistum $37 \mathrm{f}$. Dort auch über die verwandte Beerenart, eine Art Heidelbeere - empetrum nigrum (Anm. 111).

${ }^{47}$ ) Póls Saga ebd. Zum Wein aus Beeren vgl. auch: GHM 1, 230, 240, 386.

${ }^{48}$ ) Póls Saga, ebd. Unsere Kenntnis von König Sverri stammt zu einem großen Teil aus der lebendigen Lebenssschilderung der Sverris-saga (um 1300), ed. G. Indrebrö, Oslo 1920, ND Oslo 1981 ; eine Übers. des Altnord. Textes bietet J. Sephtor, Sverrissaga: The Saga of King Sverri of Norway, London 1899 (Northern Lib. 4). Zu Sverri, der als fähiger Krieger die Macht in Norwegen errang und sie im scharfen Konflikt mit dem Klerus behauptete, vgl. Zorn, Staat und Kirche, 111-149 und H. Koht, Kong Sverre, Oslo 1952.

${ }^{49}$ ) Sverrissaga, ed Ingebö, 110f. Die deutsche Übersetzung bei O. A. Johnsen, Norwegische Wirtschaftsgeschichte, Jena 1939, 115. Obwohl die Abfassung der Sverrissaga deutlich nach den Ereignissen liegt - vgl. Anm. 48 -, habe ich den anschaulichen Text hier zitiert, weil der Effekt des Alkoholhandels durch zeitgenössische Quellen bestätigt wird - vgl. die folgende Anm.

${ }^{50}$ ) Anonymus de profectione Danorum in terram sanctam aus den $1190 \mathrm{er}$ Jahren, ed. J. Lange bek, Scriptores Rerum Danicarum Medii Aevi V, Kopenhagen 1783, ND Nendeln/Liechtenstein 1969, 341-362, 353: Hic est civitas regionis illius ementiori 
So mochten Sverris Bemühungen um Beerenwein auch handelspolitische Motive gehabt haben, allein es half letztlich nichts. Das Verbot Gregors IX., Bier in der Eucharistie zu verwenden, betraf auch den Beerenwein, da es nur Wein aus Trauben - vinum de uvis - zulie $\beta^{51}$ ). Zum Handel und zur Einbindung in den europäischen Handel gab es keine Alternativen. Selbst die Erlaubnis, alternativ Bier zu verwenden, hätte die entlegenen Inseln im Nordmeer ja nicht von der Importnotwendigkeit entbunden. Sie hatten ja auch keine Rohstoffe, ihr Bier zu brauen ${ }^{52}$ ). Diese Feststellung gilt unter dem Vorbehalt, daß der Bericht über die Bierquellen, von deren Vorkommen auf Island der norwegische Königsspiegel berichtet, und deren Wasser dem Bier nach Wirkung und Geruch sehr ähnlich sei, tatsächlich kritisch zu lesen ist $^{53}$ ). Getreide, zumeist Gerste, mitunter auch Hafer und Weizen war für das Brauereiverfahren unerläßlich ${ }^{54}$ ). Der Getreidemangel aber bildete Norwegens zentrales wirtschaftliches Problem im 13. Jahrhundert. Die historische Forschung hat diesem Problem einige Aufmerksamkeit gewidmet, und es hat darum eine zum Teil heftige Auseinandersetzung gegeben. Eine Auseinandersetzung, die sich vor allem um die Rolle der Hanse in Norwegen drehte $^{55}$ ).

potentia glorisior, decorata castro regio, reliquiis sanctarum virginum adornata ... Affluentiam navium \& hominum undique venientium, Islandos, Gronlandos, Anglicos, Theotonicos, Danos, Suecos, Gutlandos, caeterasque nationes, quas dinumerare longum est ... In cunctis illius regni civitatibus uniformis consuetudo, sed vitiosa, inolevit, scilicet iugis ebrietas, quae frequenter pacis foedera rumpit, \& quandoque mansuetos quosque ad flagitia crudelitatis instigat: atque pro ioco reputantur ibidem facta criminalia. Haec etiam agit illa sine mensura potus ingurgigatio, quod cives \& advenae furore vini commoti, rapientes arma concurrant ad scelera, nec ab effusione sanguinis innocentum manus nefarias cohibere curant ... Zu Bergen vgl. auch: Bergen, Handelszentrum des beginnenden Spätmittelalters, Referate und Diskussionen d. Hans. Symposiums in Bergen vom 9. bis zum 11. Sept. 1970, hg. vom hans. Geschichtsverein, Köln-Wien 1971 (Quellen u. Darst. z. Hans. Gesch. NF 17).

${ }^{51)}$ DN I, nr. 16.

52) Vgl. zu Rohstoffen und Handel: P. Nörlund, Wikingersiedlungen in Grönland, ihre Entstehung und ihr Schicksal, Leipzig 1937; B. E. Gelsing er, Icelandic Enterprise. Commerce and Economy in the Middle Ages, Columbia 1981.

53) R. Mei ssner, Der Königspiegel, Konungsskuggsjá, Halle/S. 1944, 68.

54) Vgl. E. Plümer, Bier und Brauwesen, LMA II, München und Zürich 1983, $135-40$.

${ }^{55}$ ) Zur Zusammenfassung dieser Diskussion, die im wesentlichen zwischen der norwegischen und der deutschen Forschung ausgetragen wurde und bei der bei unbefangener Prüfung der jeweils erhebliche Einfluß wirtschaftlich/politischer Modelle auffält - eine Folge der für die Wirtschaftsgeschichte eher spärlichen Quellenlage dieser Frühzeit: J. A. Gade, The Hanseatic Control of Norwegian Commerce during the 
Denn die norddeutschen Kaufleute, die sich damals zu einem flexiblen und effektiven Verband zusammenschlossen, der erst später den Namen Hanse erhielt, hatten nach der Erschließung des Baltikums auch damit begonnen, ihren Handel mit Norwegen zu intensivieren. Ihr Anlaufhafen war hier in erster Linie Bergen, und der Handel erreichte unter König Håkon Håkonarson in den 40er Jahren des 13. Jahrhunderts einen ersten Höhepunkt. ${ }^{56}$ )

Die Kaufleute aus Westfalen und vor allem aus Lübeck, die durch ihre Verbindungen die enormen Getreidereservoirs des Baltikums für den Handel erschließen konnten, waren zunehmend an die Seite der englischen Kaufleute getreten. Sie begannen seit der Mitte des Jahrhunderts schließlich, den norwegischen Getreidehandel zu dominieren. Um 1247 sah sich Håkon angesichts stockender Lieferungen gezwungen, an die Lübecker zu appellieren: Mittatis ergo ad nos naves vestras in estate more solito cum rebus nostro regno necessariis, cum blado et brasio, et iddem nostris mercatoribus licenciam emendi concedatis, dum caristia in regno nostro duraverit ${ }^{57}$ ). Aber auch die Lübecker brachten nicht nur Getreide, wie klar wird, wenn Håkon fortfährt: Cerevisiam vero lybicensem nequaquam volumus nostri deferant mercatores, nisi quantum eis in itinere sufficiat ad bibendum, quia terra nostra in hac minime emendatur ${ }^{58}$ ). Daß die Zunahme der christlichen

Late Middle Ages, Leiden 1951 ; M. We tki, Studien zum Hanse-Norwegen Problem, in: HGBLL 70 (1951), 34-83; J. Schreiner, Bemerkungen zum Hanse-Norwegen Problem, in: HGBLL 72 (1954), 64-78; K. Helle, Neueste norwegische Forschungen über deutsche Kaufleute in Norwegen, in: HGBLL 98 (1980), 23-38; V. Henn/A. Nedkvitne, Norwegen und die Hanse, Wirtschaftliche und kulturelle Aspekte im europäischen Vergleich, Frankfurt/M. 1994 (Kieler Werkstücke, Reihe A, 11)

${ }^{56}$ ) Zur Hansegeschichte: Ph. Dollinger, Die Hanse, 4. Aufl. Stuttgart 1989; K. Friedland, Die Hanse, Stuttgart 1991; A. v. Brandt, Die Hanse als mittelalterliche Wirtschaftsorganisation, in: Die Deutsche Hanse als Mittler zwischen Ost und West, Köln-Opladen 1963 (Wiss. Abh. d. AG f. Forsch. d. Landes NRW 27), 9-37; Zum Bergenhandel dieser Zeit: K. Friedland, Kaufmannsgruppen im frühen Hansisch-Norwegischen Handel, in: Bergen (wie Anm. 50), 41-55; K. Helle, Die Deutschen in Bergen während des Mittelalters, in : Det Hanseatiske Museums Skrifter, Bergen 24 (1982), 12-29; vgl. auch: Ders., Trade and shipping between Norway and England in the reign of Håkon Håkonsson (1217-1263), in: Sjöfartshist. arbok 1967 (Norwegian Yearbook of maritime history, Bergen), 7-34; B. E. Gelsinger, A Thirteenth-century Norwegian-Castilian Alliance, in: Medievalia et Humanistica NS 10 (1981), 55-80.

57) Codex Diplomaticus Lubecensis, Lübeckisches Urkundenbuch (LUB) I. 1, Lübeck 1843, nr. 153; vgl. auch ebd., nr. 154 u. 157.

58) Ebd., nr. 153. 
Seefahrt solche Probleme auch andernorts zeitigte, ist für das Baltikum etwa aus einer livländischen Quelle belegt ${ }^{59}$ ).

So hinderte der Getreidemangel die Norweger, Isländer und Grönländer wohl in der Regel daran, ihr Bier selbst zu brauen. Es ist allerdings eine Anmerkung wert, daß auch die Zeitgenossen ihre Prioritäten trotz knapper Rohstoffe mitunter anders setzten. Denn zur selben Zeit, als die Norweger in Rom wegen ihrer Biertaufe nachfragten, sandte Friedrich II. seinen deutschen Untertanen einige Direktiven, wie sie sich angesichts der Bedrohung durch die Tartaren verhalten sollten. Die dritte Anweisung lautete: Non fiat cervisia, sed frumentum servetur ${ }^{60}$ ).

Das norwegische Mutterland aber war wohl nach den bisherigen Erkenntnissen in jedem Fall in der Lage, einen hinreichenden Bier- und Weinimport sicherzustellen. Viele Norweger profitierten überdies von dem durch christliche Standards angeregten Handel. Denn die zahlreichen Fastentage des christlichen Kalenders erschlossen dem norwegischen Stockfischexport bedeutende nordeuropäische Märkte. Der getrocknete Kabeljau war Norwegens bedeutendstes Exportgut ${ }^{61}$ ), 1252 wird er im Zolltarif von Damme erwähnt ${ }^{62}$ ).

Der Wein in Norwegen war wohl manchen ungewohnten Prüfungen ausgesetzt - so gefror König Håkon einmal bei einem winterlichen Ritt sämtlicher mitgeführter Wein - aber er war wohl auf dem Festland generell erhältlich ${ }^{64}$ ). Hier traf Thomas von Aquins Argument von den Möglichkeiten des Handels zu. Mitunter wohl im Übermaß. Aber das Problem war von den Randzonen des norwegischen Erzbistums ausgegangen, und hier wurde es nicht gelöst. Island und Grönland wurden bis in das 15. Jahrhundert hinein nicht in ein verläßliches europäisches Handelsnetz eingeschlossen und waren auf die schwer kalkulierbaren, gelegentlichen Passagen von und nach

$\left.{ }^{59}\right)$ Livländische Reimchronik vom Ausgang des 13. Jahrhunderts, ed. L. Meyer, Livländische Reimchronik, Paderborn 1876: beide mete und wîn/die Kouflûte schenketen dô/den heiden und waren vrô (V. 196-198).

60) MGH, Constitutiones et Acta publica II, ed. L. Weiland, Hannover 1896, nr. 335.

${ }^{61)} \mathrm{Vgl}$. etwa Helle, Neueste norwegische Forschungen, 34-37; Gelsinger, A Thirteenth-century alliance, 56; Schreiner, Bemerkungen, 67.

${ }^{62}$ ) Hansisches Urkundenbuch I, ed. K. Höhlbaum, Halle 1876, nr. 432, S. 145 .

${ }^{63}$ ) Zum gefrorenen Wein vgl. Hakonarsaga, ed. G. Vigfus s on, London 1887 (Icelandic Sagas II), Kap. 113. Eine englische Übersetzung des altnord. Textes in derselben Reihe: Icelandic Sagas IV: The Saga of Hacon, transl. G. W. Dasent, London 1894, Kap. 113. Die Kapitelaufteilung ist identisch. 
Norwegen angewiesen ${ }^{64}$ ). Man mußte sich weiter in den engen Spielräumen behelfen, die die liturgischen Vorschriften dem kargen Land ließen. So erhielt der Bischof von Gardar auf Grönland 1308 von seinem Amtsbruder in Bergen ein $\mathrm{Faß}$ mit Rosinen ${ }^{65}$ ). Aus diesen getrockneten Weintrauben ließ sich und läßt sich noch heute in Grenzlagen der Meßwein für die Feier der Eucharistie bereiten, indem man sie mit Wasser versetzt und schließlich preßt $\left.{ }^{66}\right)$.

Es ist wohl nicht verwunderlich, daß das sagenhafte Vinland, das der norwegischen und isländischen Überlieferung zufolge noch jenseits von Grönland lag, der Legende nach nicht nur Getreide hervorbringen sollte, sondern auch Rebstöcke mit bestem Wein ${ }^{67}$ ). Es ist aber doch verwunderlich, daß man sich in Island und Grönland angesichts der Schwierigkeiten und der Entfernung von Rom die kurialen Verbote überhaupt zu Herzen nahm und $\mathrm{da} ß$ man die römischen Standards so hoch schätzte, daß man selbst in so ungewöhnlichen Problemfällen wie der Biertaufe römischen Rat suchte. Ohne die norwegische Anfrage wäre der unerhörte Vorfall ja niemals zur Kenntnis des Papstes gelangt. Dieses Kommunikationsproblem, das zu lösen zu den Grundaufgaben jeglicher Autoritätsausübung gehört, war Innozenz IV., dem Nachfolger Gregors IX., durchaus bewußt. Nur zwei Jahre, nachdem Gregor den Erzbischof von Trondheim über die strikte römische Linie instruiert hatte, gehörte ein isländischer Vorfall zu den ersten Amtsgeschäften Innozenz' IV. Bei dieser Gelegenheit bemerkte er zur Problematik der Peripherie: Unde cum insula ipsa sit in tam extremis mundi partibus constituti quod inde nulli aut rarissimi ad apostolicam sedem accedunt..${ }^{68}$ ).

Eine solch schwache Kommunikationsstruktur bot auch Schutz vor unliebsamen Regeln. Im Falle seiner Haltung zur Verheiratung profitierte der Norden ja ganz offensichtlich von dem schwachen Kenntnisstand in Rom. Dies ist das eigentlich Kennzeichnende der gesamten Situation, und dieses

\footnotetext{
${ }^{64)} \mathrm{Vgl}$. etwa: Johannes son, A History of the Old Icelandic Commonwealth, Islendinga Saga, Manitoba 1974 (Univ. of Manitoba Icelandic Stud. 2), 222. Zu einer Übersicht über die mittelalterliche Entwicklung des isländischen Handels vor allem Gelsinger, Icelandic Enterprise.

65) GHM 3 (o. Anm. 45), 99.

${ }^{66}$ ) Vgl. Nottarp, Grönlandbistum, 39f. mit Anm. 115a.

${ }^{67}$ ) Adam von Bremen, Hamburgische Kirchengeschichte IV, 39, ed. B. Schmeidler, 3. Aufl. Hannover-Leipzig 1917 (Script. Rer. germ.), 275: Preterea unam adhuc insulam recitavit a multis in eo repertam oceano, quae dicitur Winland, eo quod ibi vites sponte nascantur, vinum optimum ferentes.
}

68) DN VII, ed. C. R. Unger/H. J. Huitfeldt, Christiania 1867, nr. 15. 
Moment unterscheidet das norwegische Problem auch grundsätzlich von den Erfahrungen der heutigen kritischen Kirche: Obwohl die Normen ihrer Kultur fremd waren, ja ihre Umsetzung auf tatsächliche, ernsthafte Probleme stieß, orientierten sich die Gläubigen im Norden der bewohnbaren Welt aus eigener Entscheidung an den römischen Vorgaben. Diese Orientierung finden wir zur selben Zeit auf kulturellem und politischen Gebiet in ähnlicher Weise ${ }^{69}$ ). Wir können das deutliche Bestreben erkennen, Norwegen stärker in die europäische Entwicklung einzubinden, ohne dabei die eigenen Freiräume aufzugeben. Schlaglichtartig wird das Vertrauen, daß man im Norden auf die römische Zentrale setzte, in einem Anliegen deutlich, das der Erzbischof von Trondheim gemeinsam mit seinem Kapitel dem Papst wenige Jahre nach der Anfrage wegen der Biertaufe vortrug. Da die Privilegien der Trondheimer Kirche durch die Lagerung ernsthaften Schaden zu nehmen drohten, baten die Kirchenleute aus dem Norden darum, zur Sicherung ihrer Rechte Abschriften der wichtigen Urkunden an der Kurie hinterlegen zu dürfen ${ }^{70}$ ). Durch diese Maßnahme, die den 50 Tagesreisen entfernten Vatikan als eine Art europäisches Archiv verstand, erhofften das norwegische Domkapitel einen Sicherheitsgewinn Es war diese hohe Wertschätzung des römischen Standards, die der Rezeption römischer Normen den Weg bereitete, und die dazu führte, daß man im Norden dabei auch Regeln übernahm, die der eigenen Situation im Grunde fremd waren.

${ }^{69}$ ) Das Problem der norwegischen Integration in Europa möchte ich an anderer Stelle dezidierter aufgreifen, zu politischen und kulturellen Einflüssen vgl.: K. Helle, Norge blir en stat (1130-1319), Handbok i Norges historie 3, 2. Aufl. Bergen-OsloTromsö 1974 (Scandinavian Univ. Books) und: H. G. Leach, Angevin Britain and Scandinavia, Cambridge/Mass. 1921, ND New York 1975 (Harvard Stud. in Compar. Lit. VI).

${ }^{70)}$ D. N. I, nr. 45. 